\title{
Infrared and Raman microspectrometries: In the near future, will have nothing to hide!
}

ince its discovery in 1928, the Raman effect has been recognized as a powerful analytical tool. In the 1970's Jobin-Yvon has coupled a Raman spectrometer to an optical microscope known as the Mole leading to a lateral resolution of 1 micron (the mole). This arrangement has now been updated: a holographic notch filter is used to reject the exciting light more efficiently and the TV camera detector has been replaced by a CCD array detector.

During the early development of IR microscopes, $\mathrm{KBr}$ lens were used which limited their use because of optical abberations. During the 50's Cassegrainian type optical systems were brought into use. In the early 1980's a resurgence in the use of infrared microspectrometry occured for two reasons: firstly due to the advantages of Fourier Transform as opposed to dispersive instrumentation and secondly due to the trends in technology. Examples of applications for both microscopies are given in papers by J.M. Chalmers and N.J. Everall.; G. Ellis, M. Gomez and C. Marco; C. Sammon, C. Mura, P. Eaton and J. Yarwood; T. Jawhari.

When comparing Infrared and Raman microspectrometry, one can say that the best spatial resolution that can be obtained in infrared is in the order of 10-20 microns and about 1 micron in the case of Raman. There is no need for special sample preparation when using Raman microspectrometry whereas a certain amount of patience and skill is required in IR microscopy. Unfortunately, for many materials continuous fluorescence problems associated with Raman spectroscopy have prevented broader applications of the Raman microprobe unless exciting radiation in the near infrared is used.

Current trends indicate a progressive improvement in technology and ease of use for both instruments: D. Schiering, T. Tague, J. Reffner, S. Vogel from Spectra-Tech (USA). In infrared, new development of different objectives (for example an ATR diamond accessory surface investigation) and in Raman, development of a confocal configuration (better depth resolution): B. Lenain from Kaiser Optical Systems (France) and a larger choice in laser exciting radiation (from UV to improve $\mathrm{X}$ and Y spatial resolution to near infrared to improve the fluorescence problems) are the main recent state of the art issues regarding IR and Raman instruments: Nguyen Quy Dao and L. Delaigue; Cl. Coupry.

Certain real novel development in IR and Raman microscopieshave recently come to light: the coupling of FTIR with DSC: R.A. Spragg from Perkin-Elmer; the improvement in data collection using an optical fibre-bundle image-compression technique which allows imaging thousand times faster than other methods (Dr Ben-Amotz, Perdue University), Near infrared microscopy (Pfizer), Near-field optical microscopy in the infrared range (A. Piednoir) where sub-wavelength resolution is obtained as well as photothermal FTIR spectroscopy (A. Hammiche et al.) and use of synchrotron radiation as an IR source: P. Dumas, G. Larry Carr and G. Williams. Finally, it is worthe mentionning that FT Raman microprobe systems have been developed but current interest in such systems is not overwhelming due to the low sensitivity of near infrared detectors and also due to the limiting spatial resolution of 5 microns when using exciting laser in the near infrared, hence conventional dispersive Raman microscope with different exciting wavelength possibilities seems to be the best system currently available: H. Boyer and J. Oswalt from Jobin Yvon (France).

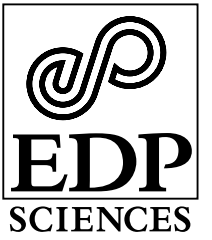

\section{Coordinated by: G. Lachenal and I. Stevenson Institut des Sciences et Techniques de l'ingénieur Laboratoire d'études des matériaux plastiques et des biomatériaux Bât. 303 et 305, 43, boulevard du 11 novembre 1918 69622 Villeurbanne Cedex, France} http://www.edpsciences.org 